\title{
Almacenamiento y flujo de carbono en suelos áridos como servicio ambiental: Un ejemplo en el noroeste de México
}

\author{
Carbon storage and flux in arid soils as an environmental service: \\ An example in northwestern Mexico
}

\author{
Fernando Ayala Niñoํㅜ, Yolanda Maya Delgado ${ }^{1}$ y Enrique Troyo Diéguez ${ }^{1 \ddagger}$
}

\footnotetext{
${ }^{1}$ Centro de Investigaciones Biológicas del Noroeste S.C. Av. Instituto Politécnico Nacional 195, Playa Palo de Santa Rita Sur. 23096 La Paz, Baja California Sur, México.

¥Autor responsable (etroyo04@cibnor.mx)
}

\section{RESUMEN}

El suelo se concibe como soporte de infraestructura y medio de producción agrícola, en tanto que los servicios ambientales que provee son poco valorados y reconocidos. Entre ellos, la captura y almacenamiento de carbono $(\mathrm{C})$ son un punto focal de interés científico por la enorme cantidad que almacena el suelo. Los objetivos de este trabajo fueron: (1) revisar los diferentes componentes, procesos y características que se presentan en los suelos áridos para el almacenamiento de C y (2) estimar el flujo y contenido de $\mathrm{C}$ en los suelos de la región semiárida de La Paz, BCS, noroeste de México, mediante dos estudios de caso. Dieciséis perfiles de suelo fueron descritos y muestreados considerando la geomorfología representativa para determinar los contenidos de carbono orgánico (COS) e inorgánico del suelo (CIS). Los valores estimados en 0.15 a $44.25 \mathrm{Mg} \mathrm{ha}^{-1}$ para COS y 0.045 a $28.19 \mathrm{Mg} \mathrm{ha}^{-1}$ para CIS, superan lo reportado para los suelos de Baja California Sur con $16 \mathrm{Mg} \mathrm{ha}^{-1}$. Valores de flujos de $\mathrm{CO}_{2}$ obtenidos de 22 meses de mediciones mensuales fueron muy variables $\left(-0.058\right.$ a $\left.0.828 \mu \mathrm{mol} \mathrm{s}^{-1} \mathrm{~m}^{-2}\right)$, valores positivos de respiración sólo se registraron tras el paso de lluvias extraordinarias. Valores negativos se asocian a la captura de $\mathrm{CO}_{2}$ a través de organismos fotosintéticamente activos como las costras biológicas del suelo, las cuales, bajo condiciones específicas de humedad y temperatura, en conjunto con los microorganismos del suelo, presentan evidencias de funcionamiento como fuente y sumidero de C.

Cita recomendada:

Ayala Niño, F., Y. Maya Delgado y E. Troyo Diéguez 2018. Almacenamiento y flujo de carbono en suelos áridos como servicio ambiental: Un ejemplo en el noroeste de México. Terra Latinoamericana 36: 93-104.

DOI: https://doi.org/10.28940/terra.v36i2.334
El presente estudio contribuye al entendimiento sobre el potencial que tienen los suelos de la región árida del noroeste de México en la provisión de SA por captura de $\mathrm{C}$ y coadyuva a incrementar el conocimiento de las CBS y su función en la relación suelo-ecosistema.

Palabras clave: carbono orgánico del suelo, carbono inorgánico del suelo, secuestro de carbono, costras biológicas.

\section{SUMMARY}

Soil is generally conceived as infrastructure support and medium for agricultural production, while the environmental services soil provides are poorly valued or recognized. Among these services, carbon (C) sequester and storage is a focal point of scientific interest due the large quantity of $\mathrm{C}$ the soil stores. The aims of this study are (1) to review the different components, processes and features that are present in dryland soils for C storage; and (2) to estimate soil $\mathrm{C}$ flux and content in the arid region of $\mathrm{La} \mathrm{Paz}$ BCS, northwestern Mexico, through two study cases. Sixteen soil profiles were described and sampled based on representative geomorphology to determine soil organic carbon (SOC) and soil inorganic carbon (SIC) contents. Estimated values of 0.15 to $44.25 \mathrm{Mg} \mathrm{ha}^{-1}$ for SOC and 0.045 to $28.19 \mathrm{Mg} \mathrm{ha}^{-1}$ for SIC surpass those reported for the soils of Baja California Sur with $16 \mathrm{Mg} \mathrm{ha}^{-1}$. Values of carbon fluxes after 22 months of monthly measurements were highly variable 
$\left(-0.058\right.$ to $\left.0.828 \mu \mathrm{mol} \mathrm{s}^{-1} \mathrm{~m}^{-2}\right)$. Positive values of soil respiration were registered only after an extraordinary rainy season; negative values may be related to $\mathrm{CO}_{2}$ capture through active photosynthetic organisms such as biological soil crusts which, under specific moisture and temperature conditions and coupled with soil organisms, exhibit evidence of their functioning as $\mathrm{C}$ source and sink. The present study contributes to understanding the potential of soils of the arid region of northwest Mexico in providing ES through carbon sequester and increases knowledge of BSC and their function in the soil-ecosystem relationship.

Index words: soil organic carbon, soil inorganic carbon, carbon sequestration, biological soil crusts.

\section{INTRODUCCIÓN}

Los servicios ambientales (SA), también conocidos como servicios ecosistémicos, son los beneficios directos o intangibles que los seres humanos obtienen de los ecosistemas; incluyen servicios de soporte (formación de suelo, ciclo de nutrientes, producción primaria), provisión (alimentos, agua de calidad y cantidad), regulación (climática, inundaciones), así como servicios culturales (espirituales, recreacionales, educativos) (MEA, 2005). Los ecosistemas han sido capaces de proveer SA para satisfacer las demandas humanas para su desarrollo; sin embargo, han sido frecuentemente subestimados, explotados, degradados y utilizados de manera no sostenible, por lo que las actividades humanas tienen un impacto significativo y creciente sobre los mismos (MEA, 2005; Chen et al., 2013). Los lineamientos internacionales establecidos en el Protocolo de Kyoto (Houghton et al., 1997), enfocados a mitigar y disminuir a largo plazo los efectos de gases de efecto invernadero (GEI) causados principalmente por $\mathrm{CO}_{2}$, han dado lugar a una política internacional dirigida a entender los procesos de generación y absorción de dichos gases, impulsando ampliamente el interés en el estudio sobre la dinámica del carbono orgánico del suelo (COS) buscando alternativas para su captura (Segura-Castruita et al., 2005).

Los suelos de los ecosistemas naturales y bajo algún tipo de manejo son un sistema regulador crítico y dinámico que genera múltiples SA. Sin embargo, la mayoría de los estudios sobre SA no consideran al suelo como un elemento clave dentro de los ecosistemas terrestres (Szabolcs, 1994), por lo que la comprensión de sus funciones y SA es aún incompleta (Swinton et al., 2006). Por ello, cabe reconocer que el SA de captura y almacenamiento de carbono $\mathrm{C}$ en el suelo es un campo de investigación en rápida expansión $\mathrm{y}$ un punto focal de interés científico a causa de la enorme cantidad de $\mathrm{C}$ orgánico almacenado en el suelo (Amundson, 2001; Chung et al., 2011). Los SA del suelo dependen de sus propiedades e interacciones, y son influenciados directamente por su uso y manejo. La erosión, salinización, disminución de contenidos de C y pérdida de biodiversidad llevan a la degradación de los suelos, lo que se convierte en un desafío global para la seguridad alimentaria y la sostenibilidad de los ecosistemas (Montgomery, 2010). La contribución de los suelos al bienestar humano va más allá de la producción alimentaria, por lo que pueden ser incorporados dentro del marco de SA debido a la variedad de servicios y funciones que proporcionan (Dominati et al., 2014; Adhikari y Hartemink, 2016), sin embargo, el SA de captura y almacenamiento de C en el suelo aún es poco valorado.

Comúnmente, el suelo se concibe como soporte de infraestructura y recurso para la agricultura, actividades que favorecen su aislamiento, fragmentación o compactación, que dan origen a diversos problemas como la pérdida de la biodiversidad y escasa captación o captura del C atmosférico. Los esfuerzos para determinar el almacenamiento de $\mathrm{C}$ en los suelos a escala global han contribuido al modelado del $\mathrm{C}$ terrestre; las estimaciones sobre su acumulación, distribución espacial a escala regional y su potencial de fijación y almacenamiento mejorarían significativamente nuestra comprensión sobre la captura y ciclos del C.

A la fecha, el conocimiento generado sobre la dinámica y distribución del $\mathrm{C}$ en el suelo es escaso, además se ha concentrado principalmente en ambientes de climas tropicales y templados (Batjes, 1996; Maya y Arriaga, 1996; Lal, 2002). En ecosistemas con déficit de agua a lo largo del año, como es el caso de las zonas áridas y semiáridas, el entendimiento de las fuentes, sumideros y flujos de $\mathrm{C}$ en los suelos es fundamental para conocer su funcionamiento dentro del ciclo global (Lal et al., 1999; Lal, 2004).

El propósito del presente trabajo fue describir los componentes y procesos principales que participan en la captura de $\mathrm{C}$ en los suelos como servicio ambiental, haciendo énfasis en un ecosistema árido del noroeste de México, mediante la aplicación de dos métodos ensayados en estudios de caso. 


\section{Las Zonas Áridas de México}

La aridez es una condición física que depende de la interacción de la radiación solar, temperatura, precipitación, dirección y fuerza del viento y la evapotranspiración, por lo que su comprensión e interpretación son complejos (Mosiño, 1983). En este sentido, las zonas áridas y semiáridas son aquellas áreas donde el promedio de lluvias es menor que las pérdidas por humedad a través de la evaporación y la evapotranspiración (FAO, 2004); y son percibidas como ecosistemas desolados con bajo potencial de producción (SUMAMAD, 2014). En México, la Comisión Nacional de las Zonas Áridas (CONAZA, 2016) define como zonas áridas a las superficies del territorio nacional en donde las precipitaciones son del orden de $250 \mathrm{~mm}$ anuales o menos, y como semiáridas donde la precipitación fluctúa entre 250 y $500 \mathrm{~mm}$. Dichas zonas se localizan en el norte y algunas partes del centro de México, abarcando poco más de la mitad del territorio nacional (Rzedowsky, 1978). Sin embargo, bajo el criterio del balance de humedad en el suelo y disponibilidad para las plantas, México cuenta con un déficit de humedad que oscila de moderado a muy severo y representa cerca del $61.2 \%$ del territorio Nacional (Monterroso y Gómez, 2003). Para el caso del estado de Baja California Sur (BCS), en el noroeste de México, la influencia del centro semipermanente de alta presión del Pacífico norte (CSAP) y de la corriente oceánica de California mantienen condiciones de aridez sobre el estado, ocasionando bajas precipitaciones. Sin embargo, durante la época de verano-otoño, la región se encuentra sometida a la influencia de actividad ciclónica del Pacífico Tropical, proporcionando masas de aire húmedo las cuales originan lluvias de regular intensidad (García y Mosiño, 1968).

\section{Suelos del Noroeste de México}

Además de la escasa precipitación que limita el crecimiento de las especies vegetales, los suelos de zonas áridas y semiáridas se caracterizan por tener baja fertilidad para la agricultura, escaso y lento desarrollo, textura arenosa y pedregosidad (Camargo y Esperón, 2005; García-Calderón, 2011). En BCS, cerca del 72\% de los suelos presentan limitantes físicas en profundidad o en superficie, además, $8 \%$ tienen restricciones químicas por altos contenidos en sales solubles y sólo $20 \%$ no tienen limitante alguna; dominan los suelos de baja fertilidad, con poca capacidad de intercambio catiónico y muy baja capacidad de retención de agua. Como resultado de la escasa precipitación y excesiva evapotranspiración, los suelos permanecen secos durante la mayor parte del año, con nula disponibilidad de agua (INEGI, 1995). De acuerdo con la World Reference Base for Soil Resources (WRB) (IUSS, 2007), los Arenosoles, Calcisoles, Durisoles, Gypsisoles, Leptosoles y Solonchaks, son suelos de referencia para los ecosistemas áridos y semiáridos. Sin embargo, el noroeste de México presenta un marcado contraste entre los procesos formadores de suelo, material parental, clima, vegetación y relieve a nivel regional, propiciando la existencia de grupos de suelo adicionales como Cambisoles, Regosoles, Fluvisoles y Vertisoles (Maya et al., 2011).

\section{Materia Orgánica del Suelo}

La materia orgánica del suelo (MOS) es uno de los recursos naturales más importantes y es la base de la fertilidad del suelo. La MOS incluye todos los compuestos orgánicos del suelo, de los organismos y productos de síntesis microbiana o de resíntesis química resistentes a la descomposición microbiana, contenidos en su mayoría en las sustancias húmicas (FAO, 2004). La fuente esencial de la MOS es el C fijado durante las reacciones de fotosíntesis, las cuales se reintegran al suelo como residuos de plantas y exudados de raíces. El contenido de MOS varía de menos de $1 \%$ en suelos arenosos y desérticos a $4 \%$ en los primeros $15 \mathrm{~cm}$ de suelos agrícolas minerales a más de $50 \%$ en suelos orgánicos. El funcionamiento de los suelos está influenciado en gran parte por su contenido de materia orgánica. La capacidad del suelo para almacenar nutrimentos, retener humedad, liberar gases de efecto invernadero, resistir la degradación física, química y biológica y para su productividad dependen de la calidad y cantidad de su materia orgánica.

\section{Carbono en el Suelo}

En las regiones áridas y semiáridas el almacenamiento de $\mathrm{C}$ representa más de un tercio del stock global debido principalmente a la superficie que cubren, así como por el almacenamiento a largo plazo del C orgánico (FAO, 2017). En el suelo, el almacén de $\mathrm{C}$ está determinado por dos diferentes componentes: el C orgánico (COS) y el C inorgánico 
del suelo (CIS). El primero se encuentra en forma de residuos orgánicos poco alterados de microorganismos, animales y vegetales, en forma de humus y en formas muy condensadas de composición próximas al carbono elemental (Jackson, 1964), el cual corresponde al 58\% de la de materia orgánica del suelo (MOS) (CastilloMorales et al., 2009; FAO, 2017). El CIS por su parte es especialmente abundante en las regiones áridas y semiáridas, con estimaciones de 695-930 Pg C a $1 \mathrm{~m}$ de profundidad (Batjes, 1996; Díaz-Hernández et al., 2003) y comprende diversos minerales predominantes estables, destacando la calcita $\left(\mathrm{CaCO}_{3}\right)$, dolomita $\left(\mathrm{MgCO}_{3}\right)$, aragonita $\left[\mathrm{CaMg}\left(\mathrm{CO}_{3}\right)_{2}\right]$ y siderita $\left(\mathrm{FeCO}_{3}\right)$, provenientes de la degradación de la roca madre o por procesos pedogenéticos de formación de carbonatos secundarios, que finalmente, conducen a la retención del $\mathrm{CO}_{2}$ atmosférico (Lal, 2009).

Dentro del ciclo global del C, los suelos son la mayor reserva de carbono orgánico en interacción con la atmósfera con estimaciones globales cercanas a $1500 \mathrm{Pg} \mathrm{C}$ en los primeros $100 \mathrm{~cm}$ superiores de suelo (Batjes, 1996; FAO, 2002), está reserva es cuatro veces mayor que el almacén de biomasa viva y muerta y aproximadamente tres veces más que el almacén atmosférico (Lal, 2004). A nivel nacional existen estudios sobre el contenido y distribución del carbono orgánico del suelo (COS) bajo diferentes enfoques y escalas de aproximación, realizados principalmente en zonas templadas y tropicales. Sin embargo, en las zonas áridas y semiáridas de México las estimaciones sobre los contenidos de COS y CIS son aún escasas (Cuadro 1).

\section{Costras Biológicas del Suelo}

Las costras biológicas de suelo (CBS) se encuentran en la superficie del mismo en la mayoría de los ecosistemas áridos y semiáridos alrededor del mundo y son uno de los componentes bióticos más importantes de estas áreas. Son comunidades que se desarrollan dentro y sobre pocos milímetros de la superficie y están formadas por organismos como cianobacterias, hongos, líquenes, musgos y briofitas, que están íntimamente asociados con las partículas del suelo (Belnap y Lange, 2003). Las CBS están ampliamente distribuidas en diversos ambientes (Belnap et al., 2003), aunque son particularmente dominantes en los ambientes de baja productividad como las zonas áridas y semiáridas, logrando alcanzar hasta un $70 \%$ de la cobertura en el suelo (Belnap y Lange, 2003).

Cuadro 1. Estudios sobre el contenido de COS y CIS en México.

\begin{tabular}{|c|c|c|}
\hline Referencia & Escala & Ecosistema/uso de suelo \\
\hline González-Molina et al., 2008 & Microcuenca & Bosque / uso agrícola \\
\hline Bojórquez Serrano et al., 2015 & Cuenca & Bosques y pastizales / uso agrícola \\
\hline Acevedo et al., 2015 & Local & Campo agrícola \\
\hline Avilés-Hernández et al., 2009 & Local & Bosque \\
\hline Solis Hernández et al., 2014 & Local & Bosque de coníferas \\
\hline Verduzco-Heredia et al., 2008 & Local & Subtropical semiárido / agrícola \\
\hline Covaleda et al., 2016 & Estatal & Bosques y selvas \\
\hline Návar-Chaidez, 2008 & Estatal & Matorral tamaulipeco \\
\hline Vela-Correa et al., 2012 & Regional & Bosques \\
\hline Luis-Mejía et al., 2007 & Regional & Bosque \\
\hline Velarde et al., 2011 & Regional & Manglar \\
\hline Cruz-Flores y Etchevers-Barra, 2011 & Nacional & Bosques templados / ANP \\
\hline Galicia et al., 2016 & Nacional & Bosques templados \\
\hline Montaño et al., 2016 & Nacional & Árido y semiárido \\
\hline Paz et al., 2016 & Nacional & Regiones ecológicas mayores \\
\hline Paz y Etchevers, 2016 & Nacional & Regiones ecológicas \\
\hline Segura-Castruita et al., 2005 & Nacional & Vegetación primaria dominante \\
\hline
\end{tabular}


Las CBS participan en procesos clave para el funcionamiento del ecosistema entre los que destaca el ciclo del $\mathrm{C}$ y nutrimentos (Delgado-Baquerizo et al., 2010; Castillo-Monroy et al., 2011). Además, la presencia de CBS tiene un notorio impacto en la modificación física del sustrato, incremento del contenido de nutrimentos, retención de agua y mantenimiento de la humedad del suelo, así como la secreción de metabolitos secundarios y formación de complejos órgano-minerales (Rivera-Aguilar et al., 2006; Bowker et al., 2011) por lo que son consideradas como elementos clave en ambientes áridos y semiáridos (Miller et al., 2011).

Durante las últimas dos décadas se ha producido un aumento sin precedentes en el interés por las CBS, lo que ha promovido importantes avances en el conocimiento de la estructura, composición y biogeografía de estas comunidades. Sin embargo, parte importante de la investigación se ha realizado en los ecosistemas áridos y semiáridos de los Estados Unidos, Australia, Israel, China y en años recientes en España (Maestre et al., 2011).

\section{Respiración del Suelo}

El estudio de la respiración del suelo es incipiente en México, donde apenas se cuenta con 28 estudios desde la última década (Cueva et al., 2016), siendo los agroecosistemas y ecosistemas forestales los que revisten mayor interés para la investigación. La respiración del suelo es una medida del $\mathrm{CO}_{2}$ liberado del suelo, resultado de la descomposición de la MOS por las comunidades microbianas y fauna del suelo, así como por la respiración de las raíces de las plantas y micorrizas asociadas (Hanson et al., 2000; GuerreroOrtíz et al., 2012). Dicho proceso es reconocido como el flujo más importante del ciclo global del C y representa cerca del $75 \%$ de la respiración total del ecosistema (Law et al., 2001); desempeña un papel fundamental en la regulación de las concentraciones de $\mathrm{CO}_{2}$ atmosférico y en la dinámica climática a escala global (Schimel, 1995). Además, constituye cerca del $25 \%$ de las emisiones globales y es uno de los principales procesos de pérdida de $\mathrm{C}$ que equivalen aproximadamente a 10 veces más que las emisiones de $\mathrm{CO}_{2}$ por combustibles fósiles y por deforestación combinadas (Raich y Schlesinger, 1992; Schimel, 1995; Conant et al., 2000; Raich et al., 2002). Después de la producción primaria bruta (cantidad de carbono total que entra al ecosistema vía fotosíntesis), la respiración del suelo es el segundo flujo más grande de C en los ecosistemas terrestres (Yuste et al., 2005). Sin embargo, a pesar de su importancia global, sólo se cuenta con un limitado entendimiento de la respiración de los suelos y sus respuestas a factores bióticos y abióticos.

La respiración del suelo es un parámetro de suma importancia para determinar la salud del ecosistema y está directamente relacionada con el soporte de raíces, macro y microorganismos, descomposición de la materia orgánica, mineralización e inmovilización de nutrimentos y actividad microbiana. Tasas elevadas de respiración de suelo indican una intensa actividad biológica, por el contrario, tasas lentas son indicativas de actividad biológica suprimida, debida al estrés inducido por un manejo inadecuado, perturbaciones climáticas o limitación de recursos (sustrato, agua, nutrimentos) para la actividad biológica (Singh et al., 2010).

\section{Cambio de Uso de Suelo}

El cambio en la cobertura y uso de suelo se encuentran en el centro de la atención de la investigación ambiental (Bocco et al., 2001), pues proporcionan la base para conocer las tendencias de los procesos de deforestación, degradación, desertificación y pérdida de la biodiversidad de una región determinada (Lambin et al., 2001). Las zonas áridas y semiáridas año con año se ven afectadas por este fenómeno resultado de las actividades humanas como la minería, el desarrollo de vías de comunicación, así como la expansión urbana, agrícola y ganadera perturbando las interacciones biológicas y los servicios ambientales (Arriaga, 2009). En los alrededores de la ciudad de La Paz, en la región árida del noroeste de México, la tendencia de los últimos años ha sido el establecimiento de asentamientos humanos con desmontes sin uso de suelo definido, o para horto-fruticultura y pastizales inducidos; dichos cambios de uso de suelo han generado desmonte acelerado del matorral sarcocaule, que es el principal tipo de vegetación que cubre planicies y lomeríos bajos de la región (Álvarez-Morales et al., 2014).

Por lo expuesto se plantearon los siguientes objetivos: 1) revisar los diferentes componentes, procesos y características que se presentan en los suelos áridos para el almacenamiento de carbono (C) y 2) estimar el flujo y contenido de $C$ en los suelos de la región semiárida de La Paz, BCS, noroeste de México, mediante dos estudios de caso. 


\section{MATERIALES Y MÉTODOS}

\section{Estudio de Caso (1): Contenidos de Carbono Orgánico e Inorgánico en los Suelos de la Región de La Paz, BCS, Noroeste de México}

Debido a la escasa información sobre el ciclo del carbono (C) y su interacción con los suelos de regiones áridas y semiáridas, se estableció un estudio referencial de los contenidos de COS y CIS almacenado en los perfiles de suelo de una región árida, de tal manera que se desarrolló un estudio en la cuenca de La Paz y parte de la cuenca de Los Planes, en el sur de la península de Baja California. El clima de la región es árido y extremadamente cálido la mayor parte del año, con temperatura media anual de $23{ }^{\circ} \mathrm{C}$, con máxima de $45^{\circ} \mathrm{C}$ en julio y agosto. La mayor radiación ocurre de abril a agosto (Troyo-Diéguez et al., 1990); la precipitación anual es de $178 \mathrm{~mm}$, con la mayor presencia de lluvias en agosto y septiembre, asociadas a tormentas tropicales. La litología es principalmente granito, granodiorita, riolita y rocas basálticas; los grupos dominantes de suelos son los Leptosoles, Regosoles, Cambisoles y Calcisoles, que son arenosos y con bajo contenido de $\mathrm{C}$ orgánico.

Se elaboró un mapa hipsométrico utilizando mapas topográficos escala 1:50 000 (INEGI, 1997), así como el modelo digital de elevación delimitado para la región de estudio (INEGI, 2014). Se describieron y muestrearon 16 perfiles de suelo con base en la geomorfología representativa dentro del área de estudio. El muestreo se diseñó tomando en cuenta la accesibilidad, así como la homogeneidad visible del terreno en cada caso, buscando sitios sin alteración aparente causada por actividades antrópicas; como criterio predefinido se buscó que los puntos a muestrear cubrieran la mayor parte del área de estudio (Figura 1). Los perfiles y sus descripciones morfológicas se realizaron hasta $100 \mathrm{~cm}$ de profundidad o hasta donde se detectase una fase física limitante. La clasificación de cada perfil se basó en la WRB (IUSS Working Group, 2015), así como en la guía para la descripción de suelos (FAO, 2006). Se colectó una muestra de alrededor de $800 \mathrm{~g}$ de suelo por horizonte en cada perfil de suelo y se transportó al laboratorio para la determinación de $\mathrm{C}$ orgánico, carbonatos $\left(\mathrm{CO}_{3}^{-2}\right) \mathrm{y}$ bicarbonatos $\left(\mathrm{HCO}_{3}^{-}\right)$. Las cantidades totales de COS y CIS se estimaron por horizonte en cada perfil de suelo mediante las ecuaciones propuestas por Batjes (1996), donde la densidad aparente $\left(\mathrm{Mg} \mathrm{m}^{-3}\right)$, la proporción de $\mathrm{C}$ orgánico e inorgánico $\left(\mathrm{g} \mathrm{kg}^{-1} \mathrm{C}\right)$, el espesor del horizonte en $\mathrm{m}$ y el contenido estimado de fragmentos de rocas $>2 \mathrm{~mm}$, fueron los parámetros para calcular los contenidos de COS y CIS por unidad de área $\left(\mathrm{Mg} \mathrm{ha}^{-1}\right)$.

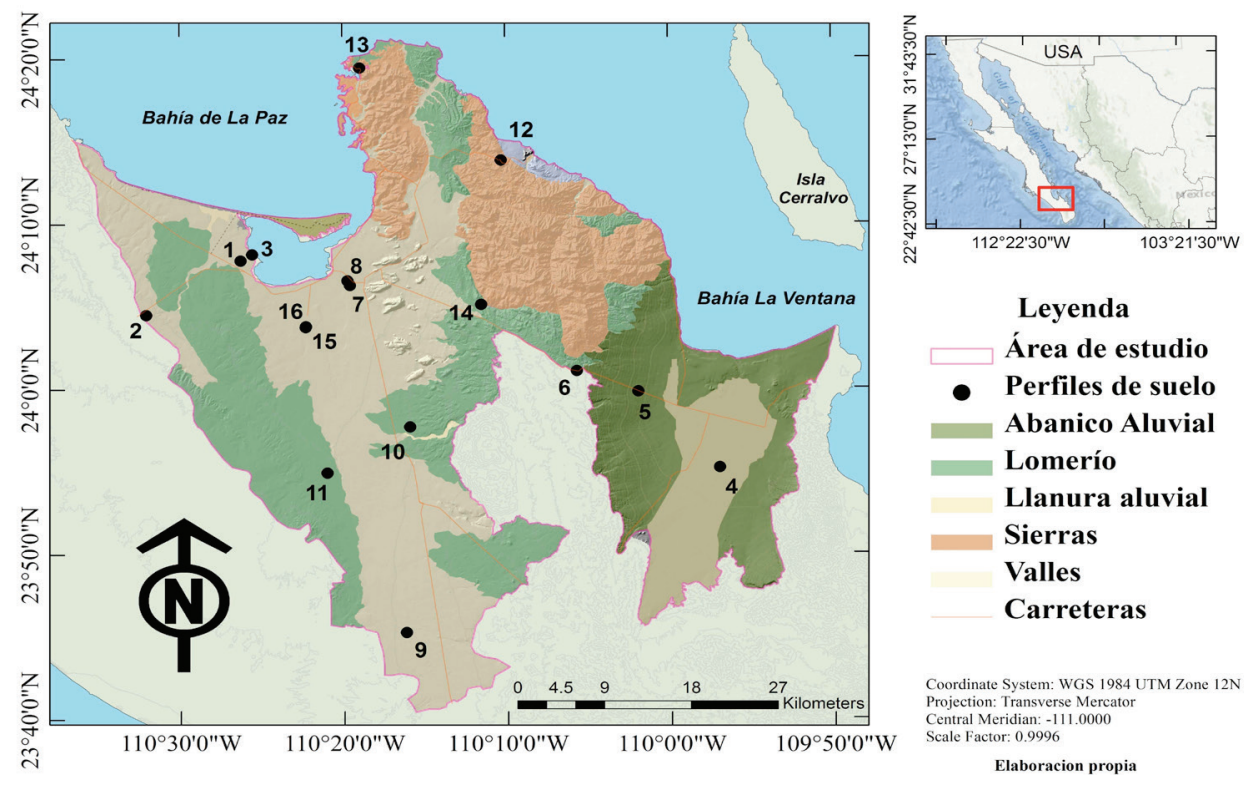

Figura 1. Mapa de las principales formas del relieve y distribución de los perfiles de suelo dentro de la cuenca de La Paz y parte de la cuenca de Los Planes al sur de la península de Baja California, México. 


\section{Estudio de Caso (2): Costras Biológicas del Suelo y Respiración Edáfica del Matorral Desértico de La Paz, BCS, Noroeste de México}

Los suelos de regiones áridas y semiáridas del noroeste de México aún no han sido cuantificados en cuanto al aporte de los flujos de $\mathrm{CO}_{2}$ hacia la atmósfera, particularmente en condiciones de estrés hídrico y altas temperaturas, tampoco por su interacción con las CBS. Para dicho propósito, se insertaron 30 collares de acero inoxidable en el suelo a $7 \mathrm{~cm}$ de profundidad distribuidos aleatoriamente en 15 cuadrantes con dimensiones $100 \times 100 \mathrm{~m}$ (dos collares por cuadrante), en la planicie aluvial dentro de la Estación Biológica del Centro de Investigaciones Biológicas del Noroeste (CIBNOR), ubicado en la porción centro-noroeste del área de estudio (Figura 1). Los collares se instalaron en sitios bajo dos condiciones de uso de suelo (20 en suelo de matorral y 10 en suelo con erosión), tomando en cuenta la presencia o ausencia de CBS, para conformar cuatro condiciones: matorral con costras (MCC), matorral sin costras (MSC), erosión con costras (ECC) y erosión sin costras (ESC). Se realizaron registros mensuales utilizando un equipo (LCpro+, ADC BioScientific Ltd., UK), para un total de 22 mediciones entre octubre de 2014 y octubre de 2016. Las mediciones se llevaron a cabo a partir de las 6:00 am hasta las 11:00 am separando el total de muestras en dos días de medición para evitar las altas temperaturas que pueden alcanzar hasta $50{ }^{\circ} \mathrm{C}$ en verano.

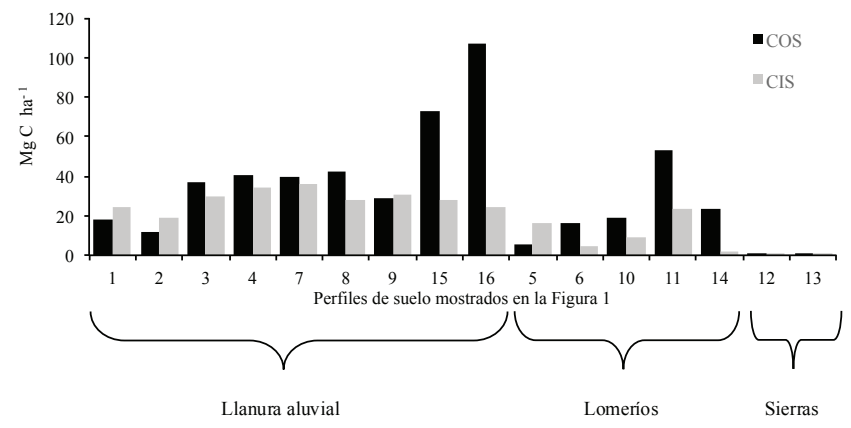

Figura 2. Contenidos de COS y CIS promedio por perfil de suelo y geomorfología representativa del área de estudio. COS: carbono orgánico del suelo; CIS: carbono inorgánico del suelo.

\section{RESULTADOS Y DISCUSIÓN}

\section{Estudio de Caso (1): Contenidos de COS Y CIS de la Región de La Paz, BCS}

Los contenidos de COS (0.15 a 44.25 $\mathrm{Mg} \mathrm{ha}^{-1}$ ) por unidad de área y unidad geomorfológica registrados (Figura 2) en los suelos de la región de estudio fueron superiores a lo reportado por Segura-Castruita et al. (2005), quienes reportaron COS de $16.0 \mathrm{Mg} \mathrm{ha}^{-1}$ para BCS a una profundidad de 0 a $20 \mathrm{~cm}$ y un valor de $24 \mathrm{Mg} \mathrm{ha}^{-1}$ para matorrales xerófilos, de acuerdo con la delimitación de la vegetación primaria dominante. En este sentido, los resultados obtenidos superaron los valores medios del COS a nivel estatal y a nivel de vegetación primaria dominante aportando información relevante sobre los contenidos de COS y CIS (la otra forma de secuestro y almacenamiento de C). Los bajos contenidos de COS son consecuencia de que las condiciones óptimas de humedad y temperatura sólo ocurren en breves periodos, de tal manera que la descomposición y mineralización de los compuestos orgánicos ocurren después de pulsos de precipitación (Arriaga y Maya, 2007). Sin embargo, de acuerdo a los resultados (Figura 2), existe una amplia variación en los contenidos de COS y CIS que puede explicarse por las diferencias en el espesor de los perfiles del suelo, que a su vez difieren en el número de horizontes. Dicha variación se relaciona asimismo con la cantidad de materia orgánica presente en cada sitio, que corresponde a la aportación de hojarasca por parte de la vegetación, al respecto, según Lal (2009) es notoria la falta de información sobre los contenidos de CIS. En este sentido, se determinaron contenidos importantes (0.045 a 28.19 $\left.\mathrm{Mg} \mathrm{ha}^{-1}\right)$ para el área de estudio.

Aunque los valores encontrados de CIS no fueron tan altos como el $\mathrm{COS}$, los carbonatos $\left(\mathrm{CO}_{3}^{2-}\right)$ y bicarbonatos $\left(\mathrm{HCO}_{3}^{-}\right)$son formas importantes de almacenamiento de $\mathrm{C}$ en las regiones áridas y semiáridas. Las llanuras y lomeríos reflejaron las medias de mayor magnitud para COS y CIS, mientras que las sierras reflejaron las medias de menor valor (Figura 2). Para el caso particular de la llanura aluvial, los sitios 9 y 10 (Figura 1), donde se detectan señales de actividades agrícolas y ganaderas, al igual que el sitio 14 ubicado en lomerío en las inmediaciones de 
la presa de La Buena Mujer, presentaron los mayores contenidos de COS y se caracterizaron por un notorio espesor, alcanzando más de $100 \mathrm{~cm}$ de profundidad. En contraste, los suelos de sierras mostraron los menores espesores de suelo y pedregosidad evidente, no alcanzando más de $4 \mathrm{~cm}$ de profundidad con muy poco o nulo contenido de COS y CIS.

Aun cuando los datos aportados por SeguraCastruita et al. (2005) muestran información a nivel nacional, su metodología utilizó la vegetación primaria dominante, usos de suelo e influencia del hombre, mientras que la metodología empleada en nuestro trabajo se basó en diferentes formas del relieve a escala de cuenca para determinar la cantidad de COS y CIS almacenado de forma más precisa, tomando como base los primeros $100 \mathrm{~cm}$ de profundidad o hasta encontrar una fase física limitante.

\section{Estudio de Caso (2): CBS y Respiración Edáfica del Matorral Desértico de La Paz, BCS}

La variación estacional y temporal de la respiración edáfica fue causada principalmente por las lluvias extraordinarias al principio del periodo de estudio, las cuales no se presentaron nuevamente en los meses posteriores (Figura 3). Valores positivos de respiración $\left(0.099\right.$ a $\left.0.828 \mu \mathrm{mol} \mathrm{s}^{-1} \mathrm{~m}^{-2}\right)$ significan un flujo de $\mathrm{CO}_{2}$ del suelo hacia la atmósfera por parte de las CBS. Por el contrario, valores negativos de respiración $(-0.058$ a $\left.0.011 \mu \mathrm{mol} \mathrm{m} \mathrm{m}^{-2} \mathrm{~s}^{-1}\right)$ significan una captura de $\mathrm{CO}_{2}$, por medio de las CBS a través de la fotosíntesis cuya composición está dominada principalmente por cianobacterias del género Microcoleus (Maya y López, 2002), las cuales pueden soportar condiciones extremas de temperatura y humedad lo que les permite ser organismos con amplia distribución en las regiones áridas y semiáridas del mundo. Por otro lado, valores positivos de respiración sólo se observaron después del paso del huracán Odile (septiembre de 2014), cuando la precipitación acumulada debida al paso del citado evento ciclónico fue de $121 \mathrm{~mm}$. En este sentido, los principales factores abióticos que determinan el proceso de la respiración del suelo son la presencia de humedad y temperatura; sin embargo, en la región árida del noroeste de México las lluvias están frecuentemente asociadas a la entrada de huracanes por el Pacífico durante el verano y lluvias ocasionales durante el invierno llamadas localmente 'equipatas' (Maya y Arriaga, 1996). De acuerdo a los resultados (Figura 3), la condición matorral con costra

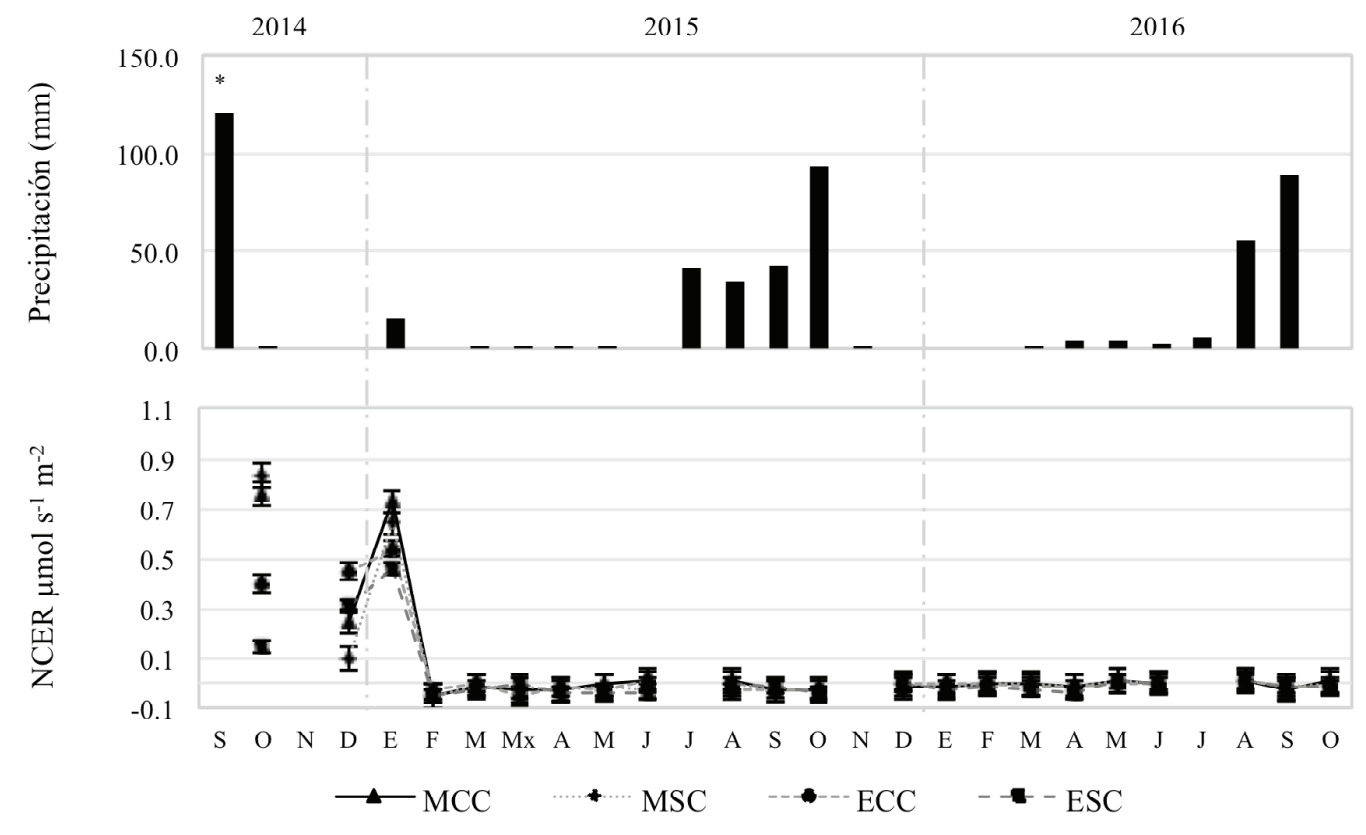

Figura 3. Precipitación mensual en la cuenca de la Paz BCS (superior) y promedio de la tasa de respiración del suelo por condición de uso de suelo y presencia/ausencia de CBS. NCER = tasa de intercambio neto de $\mathrm{CO}_{2} ; \mathrm{Mx}=$ medición extra en marzo; $\mathrm{MCC}=$ matorral con costra; $\mathrm{MSC}=$ matorral sin costra; $\mathrm{ECC}$ = erosión con costra; ESC $=$ erosión sin costra. * Huracán Odile. Barras verticales son el error estándar. 
(MCC) muestra las mayores tasas de respiración, debido principalmente a que la humedad presente en el suelo, aunado a la temperatura, logran activar los procesos metabólicos de las CBS, así como la sucesión y crecimiento de las comunidades microbianas del suelo liberando $\mathrm{CO}_{2}$ a la atmósfera. Por el contrario, la condición erosión sin costra (ESC) muestra los valores más bajos de respiración que podrían explicarse debido a la presencia en menor medida de las comunidades microbianas que habitan en el suelo, así como la escasez de raíces por la ausencia de vegetación.

Según los resultados obtenidos, la respiración edáfica puede alcanzar valores positivos después de la ocurrencia de fenómenos meteorológicos extremos, ya que durante los meses posteriores a la llegada del huracán Odile, los periodos normales de precipitación no lograron traducirse en un efecto positivo de liberación de $\mathrm{CO}_{2}$ a la atmósfera.

\section{CONCLUSIONES}

- El secuestro de carbono (C), orgánico e inorgánico, representa un importante y valioso servicio ambiental (SA) del suelo en las regiones en donde las condiciones climáticas limitan el desarrollo de la cobertura vegetal. Es por ello que en los ecosistemas de las regiones áridas el suelo es el gran sumidero de $\mathrm{C}$, a diferencia de los ecosistemas templados y tropicales en los que el C está secuestrado en la biomasa vegetal.

Aunque los contenidos de carbono orgánico (COS) en los suelos de la región estudiada resultaron bajos, superan las estimaciones realizadas a nivel estatal y a nivel de vegetación primaria dominante. Así mismo, las estimaciones de carbono inorgánico del suelo (CIS) demuestran que es una forma importante de almacenamiento que participa en los procesos de captura y retención del carbono atmosférico en el suelo, aportando un valioso servicio ambiental por captura de C.

- Las costras biológicas de suelo (CBS) participan activamente en los flujos de $\mathrm{CO}_{2}$ del suelo a la atmósfera; esto significa que existe una captura de C por parte de las CBS mediante la fotosíntesis y que sólo bajo condiciones específicas de humedad y temperatura, estas comunidades liberarán $\mathrm{CO}_{2}$ a la atmósfera, logrando una doble función en el ecosistema como fuente y sumidero de $\mathrm{C}$.

- Estudios sobre la dinámica, procesos y mecanismos del C a múltiples escalas son escasos en la región árida del noroeste de México, lo cual obedece a que la mayor parte de las investigaciones referentes al ciclo del C $\mathrm{y}$ sus interacciones se han enfocado a ecosistemas templados y tropicales.

- El presente estudio de caso generó información inédita sobre la cuantificación de los flujos de $\mathrm{CO}_{2}$ bajo la influencia de las CBS, cambios en el uso de suelo y de la variación temporal. Los resultados alcanzados contribuyen a demostrar que los suelos de la región árida del noroeste de México proveen un SA por captura de $\mathrm{C}$ y permiten incrementar el conocimiento de las CBS y su función en la relación suelo-ecosistema. Sin embargo, los beneficios se vislumbran a mediano y largo plazo con efectos potenciales en el ordenamiento del uso del suelo, aunque aún no existe un mecanismo que transforme los procesos ecológicos de captura de carbono en beneficios económicos a los propietarios de las tierras de regiones áridas.

\section{AGRADECIMIENTOS}

Los autores agradecen a Manuel Trasviña y Miriam L. de Haro Hernández del Laboratorio de Suelos del CIBNOR, por su apoyo en los análisis de laboratorio; a Álvaro González M. y Arturo Cruz F. del Laboratorio de Hidrología e Irrigación por los datos climatológicos y a Gregorio Lucero, Christian Silva, Adrián Jordán y Raymundo Ceseña por su apoyo en el trabajo de campo. Agradecemos al CONACYT por la beca doctoral otorgada (CVU 246829), así como a los revisores por los comentarios y sugerencias al manuscrito. E1 presente trabajo de investigación se realizó con apoyo financiero otorgado por el Fondo Sectorial FSID sobre el Agua CONACYT-CONAGUA (Proyecto 0249265).

\section{LITERATURA CITADA}

Acevedo, D. C., E. Hernández-Acosta, R. Maldonado-Torres, M. E. Álvarez-Sánchez. 2015. Variabilidad espacial del carbono en un suelo después de 10 años de retiro e incorporación de residuos de cosecha. Terra Latinoamericana 33: 199-208.

Adhikari, K. and A. E. Hartemink. 2016. Linking soils to ecosystem services-A global review. Geoderma 262: 101-111. doi: 10.1016/j.geoderma.2015.08.009.

Álvarez-Morales, Y., E. Troyo-Diéguez, A. Nieto-Garibay, F. A. Beltrán-Morales, B. Murillo-Amador, A. Cruz-Falcón y J. Navejas-Jiménez. 2014. Vulnerabilidad hidroclimática y degradación del suelo en regiones agrícolas semiáridas. Terra Latinoamericana 32: 47-58.

Arriaga, L. and Y. Maya. 2007. Spatial variability in decomposition rates in a desert scrub of northwestern Mexico. Plant Ecol. 189: 213-225. doi: 10.1007/s11258-006-9178-4. 
Arriaga, L. 2009. Implicaciones del cambio de uso de suelo en la biodiversidad de los matorrales xerófilos: un enfoque multiescalar. Invest. Amb. Cienc. Pol. Pub. 1: 6-16.

Amundson, R. 2001. The carbon budget in soils. Annu. Rev. Earth Planet. Sci. Lett. 29: 535-562. doi: 10.1146/annurev. earth.29.1.535.

Avilés-Hernández, V., A. Velázquez-Martínez, G. Ángeles-Pérez, J. Etchevers-Barra, H. de los Santos-Posadas y T. Llanderal. 2009. Variación en almacenes de carbono en suelos de una toposecuencia. Agrociencia 43: 457-464.

Batjes, N. H. 1996. Total carbon and nitrogen in the soils of the world. Eur. J. Soil Sci. 47: 151-163. doi: 10.1111/j.13652389.1996.tb01386.x.

Belnap, J. and O. L. Lange. 2003. Biological soil crusts: Structure, function and management. Springer-Verlag Berlin Heidelberg. Berlin.

Belnap, J., C. V. Hawkes, and M. K. Firestone. 2003. Boundaries in miniature: Two examples from soil. BioScience 53: 739-749. https://doi.org/10.1641/0006-3568(2003)053[0739:BIMTEF] 2.0.CO;2.

Bocco, G., M. Mendoza y O. R. Masera. 2001. La dinámica del cambio de uso del suelo en Michoacán. Una propuesta metodológica para el estudio de los procesos de deforestación. Invest. Geogr. 44: 18-38.

Bojórquez Serrano, J. I., L. A. Castillo-Pacheco, A. HernándezJiménez, J. D. García-Paredes y A. Madueño-Molina. 2015. Cambios en las reservas de carbono orgánico del suelo bajo diferentes coberturas. Cult. Trop. 36: 63-69.

Bowker, M. A., R. L. Mau, F. T. Maestre, C. Escolar, and A. P. Castillo-Monroy. 2011. Functional profiles reveal unique ecological roles of various biological soil crust organism. Funct. Ecol. 25: 787-795. doi: 10.1111/j.1365-2435.2011.01835.x.

Camargo-Ricalde, S. L. y M. Esperón-Rodríguez. 2005. Efecto de la heterogeneidad espacial y estacional del suelo sobre la abundancia de esporas de hongos micorrizógenos arbusculares en el valle semiárido de Tehuacán-Cuicatlán, México. Rev. Biol. Trop. 53: 339-352.

Castillo-Morales, M., G. Linares-Fleites, M. A. Valera-Pérez, N. E. García-Calderón y O. A. Acevedo-Sandoval. 2009. Modelación de la materia orgánica en suelos volcánicos de la región de Teziutlán, Puebla, México. Rev. Latinoam. Rec. Nat. 5: $148-154$.

Castillo-Monroy, A. P., F. T. Maestre, A. Rey, S. Soliveres, and P. García-Palacios. 2011. Biological soil crust microsites are the main contributor to soil respiration in a semiarid ecosystem. Ecosystems 14: 835-847. doi: 10.1007/s10021-011-9449-3.

Chen, T., G. R. Van der Werf, R. A. M. de Jeu, G. Wang, and A. J. Dolman. 2013. A global analysis of the impact of drought on net primary productivity. Hydrol. Earth Syst. Sci. 17: 3885-3894. doi: 10.5194/hess-17-3885-2013.

Chung, I. K., J. Beardall, S. Metha, D. Sahoo, and S. Stojkovic. 2011. Using marine macroalgae for carbon sequestration: A critical appraisal. J. Appl. Phycol. 23: 877-886. doi: org/10.1007/s10811-010-9604-9.

Conant, R. T., J. M. Klopatek, and C. C. Klopatek. 2000. Environmental factors controlling soil respiration in three semiarid ecosystems. Soil Sci. Soc. Am. J. 64: 383-390. doi: 10.2136/sssaj2000.641383x.
CONAZA (Comisión Nacional de las Zonas Áridas). 2016. http:// www.conaza.gob.mx/transparencia/Documents/Zonas Áridas.doc (Consulta: mayo 30, 2017).

Vela Correa, G., J. López-Blanco y M. de L. Rodríguez-Gamiño. 2012. Niveles de carbono orgánico total en el suelo de conservación del Distrito Federal, centro de México. Invest. Geog. 77: 18-30.

Covaleda, S., F. Paz y A. Ranero. 2016. Carbono edáfico en Chiapas: Planteamiento de políticas públicas de mitigación de emisiones. Terra Latinoamericana 34: 97-112.

Cruz-Flores, G. y J. D. Etchevers-Barra. 2011. Contenidos de carbono orgánico de suelos someros en pinares y abetales de áreas protegidas de México. Agrociencia. 45: 849-862.

Cueva, A., C. A. Robles-Zazueta, J. Garatuza-Payan, and E. A. Yépez. 2016. Soil respiration in México. Advances and future directions. Terra Latinoamericana 34: 253-269.

Delgado-Baquerizo, M., A. P. Castillo-Monroy, F. T. Maestre, and A. Gallardo. 2010. Plants and biological soil crust modulate the dominance of $\mathrm{N}$ forms in a semi-arid grassland. Soil Biol. Biochem. 42: 376-378.

Díaz-Hernández, J. L., E. B. Fernández, and J. L. González. 2003. Organic and inorganic carbon in soils of semiarid regions: A case study from the Guadix-Baza basin (Southeast Spain). Geoderma 114: 65-80. doi: 10.1016/S0016-7061(02)00342-7.

Dominati, E., A. Mackay, S. Green, and M. Patterson. 2014. A soil change-based methodology for the quantification and valuation of ecosystem services from agro-ecosystems: A case study of pastoral agriculture in New Zealand. Ecol. Econ. 100: 119-129. doi: 10.1016/j.ecolecon.2014.02.008.

FAO (Food and Agriculture Organization of the United Nations). 2002. Land degradation assessment in drylands-LADA project. World Soil Resources Reports No. 97. Food and Agriculture Organization of the United Nations. Rome, Italy. ISBN 92-5104797-9.

FAO (Food and Agriculture Organization of the United Nations). 2004. Carbon sequestration in drylands. Report on World Soil Resources, No.102. Food and Agriculture Organization of the United Nations. Rome, Italy. ISBN 92-5-105230-1

FAO (Food and Agriculture Organization of the United Nations). 2006. Guidelines for Soil Description. 4th ed. Food and Agriculture Organization of the United Nations. Rome, Italy. ISBN 92-5-105521-1.

FAO (Food and Agriculture Organization of the United Nations). 2017. Soil Organic Carbon the hidden potential. Food and Agriculture Organization of the United Nations. Rome, Italy. ISBN 978-92-5-109681-9.

Galicia, L., A. M. Gamboa-Cáceres, S. Cram, B. Chávez-Vergara, V. Peña-Ramírez, V. Saynes y C. Siebe. 2016. Almacén y dinámica del carbono orgánico del suelo en bosques templados de México. Terra Latinoamericana 34: 1-29.

García, E. y A. P. Mosiño. 1968. Los climas de la Baja California. pp. 29-56. In: Memoria 1966-1967 del Comité Mexicano para el Decenio Hidrológico Internacional, Instituto de Geofísica, UNAM. México, D.F.

García-Calderón, N. E. 2011. Los ecosistemas como factor geográfico de distribución de suelos. pp. 99-118. In: P. Krasilnikov, F. J. Jiménez-Nava, T. R. Trujillo, N. E. GarcíaCalderón (eds.). Geografía de suelos de México. UNAM Facultad de Ciencias. México, D. F. 
González-Molina, L., J. D. Etchevers-Barra y C. HidalgoMoreno. 2008. Carbono en suelos de ladera: Factores que deben considerarse para determinar su cambio en el tiempo. Agrociencia 42: 741-751.

Guerrero-Ortíz, P. L., R. Quintero-Lizaola, V. Espinoza-Hernández, G. S. Benedicto-Valdés y M. de J. Sánchez-Colín. 2012. Respiración de $\mathrm{CO}_{2}$ como indicador de la actividad microbiana en abonos orgánicos de Lupinus. Terra Latinoamericana 30: 355-362.

Hanson, P. J., N. T. Edwards, C. T. Garten, and J. A. Andrews. 2000. Separating root and soil microbial contributions to soil respiration: A review of methods and observations. Biogeochemistry 48: 115-146. doi:10.1023/A:1006244819642.

Solis Hernández, A., J. A. Nájera-Luna, J. Méndez-González, B. Vargas-Larreta y M. Álvarez-Gallegos. 2014. Carbono orgánico del suelo en rodales silvícolas del ejido La Victoria, Pueblo Nuevo, Durango. Invest. Cienc. 22: 5-11.

Houghton, J. T., L. G. Meira Filho, B. Lim, K. Treanton, I. Mamaty, Y. Bonduky, D. J. Griggs, and B. A. Callender. 1997. Revised 1996 IPCC guidelines for national greenhouse house gas inventory. Reference Manual Volume 3. Intergubernamental Panel on Climate Change. Ginebra, Suiza.

INEGI (Instituto Nacional de Estadística y Geografía). 1995. Síntesis Geográfica de Baja California Sur. Instituto Nacional de Estadística y Geografía. México, D. F.

INEGI (Instituto Nacional de Estadística y Geografía). 1997. Mapa Topográfico La Paz. Escala 1:250,000. Instituto Nacional de Estadística y Geografía. México, D. F.

INEGI (Instituto Nacional de Estadística y Geografía). 2014. Modelo Digital de Elevación (DEM) Baja California Sur. Instituto Nacional de Estadística y Geografía. México, D. F.

IUSS (International Union of Soil Sciences) Grupo de Trabajo WRB. 2007. Base Referencial Mundial del Recurso Suelo. Primera actualización 2007. Informes sobre Recursos Mundiales de Suelos No. 103, FAO. Roma.

IUSS (International Union of Soil Sciences) Working Group WRB. 2015. World Reference Base for Soil Resources 2014, update 2015 International soil classification system for naming soils and creating legends for soil maps. World Soil Resources Reports No. 106. FAO, Rome.

Jackson, M. L. 1964. Análisis químicos de suelos. Omega. Barcelona, España.

Lal, R. and J. P. Bruce. 1999. The potential of world cropland soils to sequester $\mathrm{C}$ and mitigate the greenhouse effect. Environ. Sci. Pol. 2: 177-185. doi: 10.1016/S1462-9011(99)00012-X.

Lal, R. 2002. Carbon sequestration in dryland ecosystem of west Asia and North Africa. Land Degrad. Dev. 13: 45-59. doi:10.1002/1dr.477.

Lal, R. 2004. Soil carbon sequestration to mitigate climate change. Geoderma 123: 1-22. doi: 10.1016/j.geoderma.2004.01.032.

Lal, R. 2009. Sequestering carbon in soils of arid ecosystems. Land Degrad. Dev. 20: 441-454. doi: 10.1002/ldr.934.

Lambin, E. F., B. L. Turner, H. J. Geist, S. B. Agbola, A. Angelsen, J. W. Bruce, O. T. Coomes, R. Dirzo, G. Fischer, C. Folke, P. S. George, K. Homewood, J. Imbernon, R. Leemans, X. Li, E. F. Moran, M. Mortimore, P. S. Ramakrishnan, J. F. Richards, H. Skånes, W. Steffen, G. D. Stone, U. Svedin, T. A. Veldkamp, C. Vogel, and J. Xu. 2001. The causes of land-use and land-cover change: Moving beyond the myths. Global Environ. Change 11: 261-269. doi: 10.1016/S0959-3780(01)00007-3.
Law, B., F. Kelliher, D. Baldocchi, P. Anthoni, J. Irvine, D. Moore, S. Van Tuyl. 2001. Spatial and temporal variation in respiration in a young ponderosa pine forest during a summer drought. Agric. For. Meteorol. 110: 27-43. doi:10.1016/S01681923(01)00279-9

Luis-Mejía, S., A. Gómez-Guerrero, J. D. Etchevers-Barra, G. Ángeles-Pérez, M. A. López-López y W. R. Horwath. 2007. Acumulación de carbono orgánico en el suelo en reforestaciones de Pinus michoacana. Agociencia 41: 711-721.

Maestre, F. T., M. A. Bowker, Y. Cantón, A. P. Castillo-Monroy, J. Cortina, C. Escolar, A. Escudero, R. Lázaro, and I. Martínez. 2011. Ecology and functional roles of biological soil crust in semi-arid ecosystems of Spain. J. Arid Environ. 75: 1282-1291. doi: 10.1016/j.jaridenv.2010.12.008.

Maya, Y. and L. Arriaga. 1996. Litterfall and phenological patterns of the dominant overstorey species of a desert scrub community in northwestern Mexico. J. Arid Environ. 34: 23 35. doi: 10.1006/jare.1996.0090.

Maya, Y. and A. López-Cortés. 2002. Cyanobacterial microbiotic crusts in eroded soils of a tropical dry forest in the Baja California Peninsula, Mexico. Geomicrobiol. J. 19: 505-518. doi: 10.1080/01490450290098469.

Maya, Y., F. R. Venegas y F. J. Manríquez. 2011. Geografía de suelos regional: Península de Baja California. pp. 217-253. In: P. Krasilnikov., F. J. Jiménez-Nava, T. R. Trujillo y N. E. García-Calderón (eds.). Geografía de suelos de México. UNAM Facultad de Ciencias. México, D. F.

MEA (Millennium Ecosystem Assessment). 2005. Ecosystems and human well-being: Our human planet. Summary for Decision Makers. Millennium Ecosystem Assessment. Island Press. Washington, DC, USA.

Miller, M. E., R. T. Belote, M. A. Bowker, and S. L. Garman. 2011. Alternative states of a semiarid grassland ecosystem: implications for ecosystem services. Ecosphere 2: 1-18. doi: abs10.1890ES11-00027.1.

Montgomery, H. L. 2010. How is soil made? Crabtree Publishing. New York, NY, USA.

Montaño, N. M., F. Ayala, S. H. Bullock, O. Briones, F. García O, S. García, Y. Maya, Y. Perroni, C. Siebe, Y. Tapia T, E. Troyo y E. Yépez. 2016. Almacenes y flujos de carbono en ecosistemas áridos y semiáridos de México: síntesis y perspectivas. Terra Latinoamericana 34: 39-59.

Monterroso, A. I. y J. D. Gómez. 2003. Delimitación de las zonas áridas, semiáridas y sub-húmedas secas de acuerdo con la metodología de Thornwaite modificada. Comisión Nacional de las Zonas Áridas y Universidad Autónoma Chapingo. Chapingo, México.

Mosiño A. P. 1983. Climatología de las zonas áridas y semiáridas de México pp. 9-36. In: Memorias del simposio "Caracterización, uso actual y potencial de los recursos agrícolas de zonas áridas y semiáridas de México". Colegio de Postgraduados, Chapingo. México.

Návar-Chaidez, J. J. 2008. Carbon fluxes resulting from land-use changes in Tamaulipan thornscrub of northeastern Mexico. Carbon Balance Manage. 3: 1-11. doi: 10.1186/1750-06803-6.

Paz, F., J. Argumedo-Espinoza, C. O. Cruz-Gaistardo, J. D. Etchevers B. y B. de Jong. 2016. Distribución espacial y temporal del carbono orgánico del suelo en los ecosistemas terrestres de México. Terra Latinoamericana 34: 289-310. 
Paz, F. y J. Etchevers. 2016. Distribución a profundidad del carbono orgánico en los suelos de México. Terra Latinoamericana 34: 339-355.

Raich, J. W. and W. H. Schlesinger. 1992. The global carbon dioxide flux in soil respiration and its relationship to vegetation and climate. Tellus 44: 81-99. doi: 10.1034/j.1600-0889.1992.t011-00001.x.

Raich, J. W., C. S. Potter, and D. Bhagawati. 2002. Interannual variability in global soil respiration, 1980-94. Global Change Biol. 8: 800-812. doi: 10.1046/j.1365-2486.2002.00511.x.

Rivera-Aguilar, V., G. Montejano, S. Rodríguez-Zaragoza, and A. Durán-Díaz. 2006. Distribution and composition of cyanobacteria, mosses and lichens of the biological soil crust of the Tehuacán Valley, Puebla, México. J. Arid Environ. 67: 208-225. doi: 10.1016/j.jaridenv.2006.02.013.

Rzedowsky, J. 1978. Vegetación de México. Limusa. México, D. F.

Schimel, D. S. 1995. Terrestrial ecosystems and the carbon cycle. Global Change Biol. 1: 77-91. doi: 10.1111/j.1365-2486.1995. tb00008.x

Segura-Castruita, M. A., P. Sánchez-Guzmán, C. A. Ortíz-Solorio y M. C. Gutiérrez-Castorena. 2005. Carbono orgánico en suelos de México. Terra Latinoamericana. 23: 21-28.

Singh, B. K., R. D. Bardgett, P. Smith, and D. S. Reay. 2010. Microorganisms and climate change: Terrestrial feedbacks and mitigation options. Nat. Rev. Microbiol. 8: 779-790. doi: 10.1038/nrmicro2439.
SUMAMAD (Sustainable Management of Marginal Drylands). 2014. Drylands. Sustaining Livelihoods and Conserving Ecosystem Services. The United Nations University. Ontario, Canada.

Swinton, S. M., F. Lupi, G. P. Robertson, and D. A. Landis. 2006. Ecosystem services from agriculture: looking beyond the usual suspects. Am. J. Agric. Econ. 88: 1160-1166. doi: 10.1111/j.1467-8276.2006.00927.x.

Szabolcs, I. 1994. The concept of soil resilience. pp. 33-39. In: D. J. Greenland and I. Szabolcs (eds.). Soil resilience and sustainable land use. CAB International. Wallingford, UK.

Troyo-Diéguez, E., F. de Lachica-Bonilla, and J. L. FernándezZayas. 1990. A simple aridity equation for agricultural purposes in marginal zones. J. Arid Environ. 19: 353-362.

Velarde, V. E., J. I. Valdez-Hernández, V. M. Ordaz-Chaparro, J. F. Gallardo-Lancho, J. Pérez-Nieto y C. Ayala-Sánchez. 2011. Evaluación del carbono orgánico en suelos de los manglares de Nayarit. Rev. Mex. Cienc. For. 2: 47-58.

Verduzco-Heredia, M. A., M. T. Hernández-del Real, M. SánchezHeráldez y C. Morán-Rodríguez. 2008. Influencia de la temperatura, humedad y labranza en el carbón orgánico de suelos agrícolas subtropicales semiáridos. Rev. Latinoam. Recur. Nat. 4: 229-238.

Yuste, J. C., M. Nagy, I. A. Janssens, A. Carrara, and R. Ceulemans. 2005. Soil respiration in a mixed temperate forest and its contribution to total ecosystem respiration. Tree Physiol. 25: 609-619. 